\title{
Riesgos y complicaciones del uso a largo plazo de los inhibidores de la bomba de protones
}

\author{
Risks and complications of long-term use \\ of proton pump inhibitors
}

\author{
Enrique Cervintes-Pérez ${ }^{1,2 *}$, Gabino Cervintes-Guevara ${ }^{2,3}$, Lorena A. Cervantes-Pérez ${ }^{4}$, \\ Gabino Cervantes-Pérez ${ }^{3}$, Guillermo A. Cervantes-Cardona ${ }^{5}$ y Sol Ramírez-Ochoa ${ }^{6}$
}

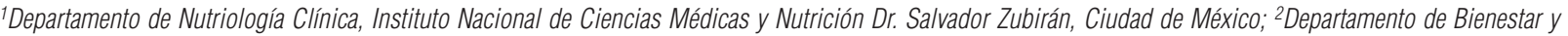
Desarrollo Sustentable, Centro Universitario del Norte, Universidad de Guadalajara, Colotlán, Jal.; ${ }^{3}$ Servicio de Gastroenterología, Hospital Civil de Guadalajara Fray Antonio Alcalde, Guadalajara, Jal.; ${ }^{4}$ Unidad de Trasplante de Médula Ósea, Instituto Nacional de Cancerología, Ciudad de México; ${ }^{5}$ Departamento de Disciplinas Filosófico, Metodológicas e Instrumentales, Centro Universitario de Ciencias de la Salud, Universidad de Guadalajara, Guadalajara, Jal.; ${ }^{\circ}$ Servicio de Medicina Interna, Hospital Civil de Guadalajara Fray Antonio Alcalde, Guadalajara, Jal. México

\begin{abstract}
Proton pump inhibitors (PPIs) are among the most commonly used medications in the world. PPIs suppress gastric acid secretion, and they are widely considered as the medications of choice for the treatment of acid-peptic disorders, Helicobacter pylori infection, and for the prevention of nonsteroidal anti-inflammatory drug-induced peptic ulcers. There are well-established side effects such as headache, diarrhea, constipation, and abdominal pain. In recent years, several studies have been published describing numerous putative, serious, warning side effects, raising concern among the medical community, including hypomagnesemia, vitamin $\mathrm{B}_{12}$ deficiency, bacterial overgrowth, risk of fractures, Clostridioides difficile infection, chronic and acute kidney disease, dementia, community-acquired pneumonia, cardiac diseases and a potential carcinogenic role. These findings have led to subsequent investigations to asses these potential risks in patients treated chronically with PPIs. However, much of this evidence is inadequate to establish a causal association between PPIs use and the risk for development of serious adverse effects.
\end{abstract}

Key words: Proton pump inhibitors. Gastroesophageal reflux disease. Peptic ulcer disease. Chronic use. Accepted clinical indications. Long-term risk.

\section{Resumen}

Los inhibidores de la bomba de protones (IBP) se encuentran entre los medicamentos más utilizados en todo el mundo. Los IBP se encargan de suprimir la secreción de ácido gástrico y son ampliamente considerados como los medicamentos de elección para el tratamiento de los desórdenes ulcerosos pépticos, la infección por Helicobacter pylori y para la prevención de las úlceras pépticas asociadas al uso de antiinflamatorios no esteroideos. Existen efectos secundarios bien conocidos tales como cefalea, diarrea, estreñimiento y dolor abdominal. En los últimos años se han publicado numerosos estudios describiendo efectos secundarios serios y graves causando alarma y controversia en la comunidad médica, incluyendo hipomagnesemia, deficiencia de vitamina $B_{12}$, sobrecrecimiento

\section{Correspondence:}

*Enrique Cervantes-Pérez

E-mail: enrique19896@hotmail.com
Date of reception: 07-09-2019

Date of acceptance: 18-05-2020

DOI: 10.24875/HMCM.20000228
Available online: $09-07-2020$

Hosp Med Clin Manag. 2020;13:63-70

2604-0018 / @ 2020 Mexican Regional Hospitals of High Specialty and Federal Hospitals. Published by Permanyer. This is an open access article under the CC BY-NC-ND license (http://creativecommons.org/licenses/by-nc-nd/4.0/). 
bacteriano, fracturas óseas, infección por Clostridioides difficile, enfermedad renal aguda y crónica, demencia, neumonía adquirida en la comunidad, enfermedades cardiacas, así como un potencial rol carcinogénico. Estos hallazgos han llevado a realizar estudios para valorar estos riesgos en pacientes que utilizan IBP de manera crónica. Sin embargo, gran parte de la evidencia es inadecuada para establecer asociación de causa entre el uso de IBP y el riesgo para desarrollar dichos efectos adversos severos.

Palabras clave: Inhibidores de la bomba de protones. Enfermedad por reflujo gastroesofágico. Enfermedad por ulcera péptica. Uso crónico. Indicaciones clínicas aceptadas. Riesgo a largo plazo.

\section{INTRODUCCIÓN}

Los inhibidores de la bomba de protones (IBP) se encuentran entre los medicamentos más utilizados en todo el mundo ${ }^{1}$. Tan solo en EE.UU., más del $7 \%$ de la población adulta utiliza IBP de manera prescrita y, sin duda alguna, muchos más los usan sin prescripción médica ${ }^{2}$. Estos fármacos se encargan de suprimir la secreción de ácido gástrico y son ampliamente considerados como los medicamentos de elección para el tratamiento de desórdenes tales como enfermedad por reflujo gastroesofágico y enfermedad ulcerosa péptica, así como para la prevención y control de síntomas asociados a esófago de Barret, profilaxis de sangrado asociado al uso de antiinflamatorios no esteroideos (AINE) y para patologías hipersecretoras como el síndrome de Zollinger-Ellison (Tabla 1). Existen efectos secundarios bien establecidos de los IBP, tales como cefalea, diarrea, estreñimiento y molestias abdominales leves, relativamente poco comunes y fáciles de manejar. Sin embargo, en años recientes se han publicado diversos estudios describiendo efectos secundarios serios y graves, lo que ha causado alarma tanto en pacientes como en la comunidad médica. La mayoría de estas investigaciones fueron retrospectivas, observacionales, con métodos estadísticos aceptados, y revelaron una asociación general de leve a moderada, pero sin demostrar causa y efecto ${ }^{3}$.

\section{ASOCIACIONES DESCRITAS}

La hipomagnesemia, una alteración descrita desde el año 2006 en pacientes tomando IBP por más de un año, se presenta con espasmo carpopedal. Lo anterior quedó de manifiesto en una revisión de nueve estudios observacionales con 109,798 pacientes donde se reportó un incremento del $43 \%$ del riesgo de hipomagnesemia en pacientes con IBP, sugiriendo una asociación causal ${ }^{4}$.
También se encontró una relación entre la deficiencia de vitamina $B_{12}$ y el uso crónico de IBP cuando se compararon 25,956 pacientes con deficiencia de dicha vitamina, frente a 184,199 pacientes sanos para valorar la asociación con la terapia de supresión de ácido gástrico. Aquellos que recibieron IBP por más de dos años tuvieron un incremento del $65 \%$ en el riesgo de deficiencia de vitamina $B_{12}$ al compararse con los no usuarios $^{5}$. Lo, et al. incluyeron 11 estudios y encontraron un incremento en el riesgo de desarrollar sobrecrecimiento bacteriano en el intestino delgado entre quienes tomaban regularmente IBP comparado con los no usuarios. Este riesgo fue más de siete veces mayor en aquellos estudios que utilizaron aspirado duodenal/yeyunal para el diagnóstico ${ }^{6}$. Otros trabajos han encontrado también incremento en el riesgo de fracturas en pacientes que han usado de manera crónica IBP. El resultado de 18 pruebas observacionales reportó que el uso de IBP se asoció con un aumento del $33 \%$ en el riesgo de fracturas en cualquier sitio, del $26 \%$ en el riesgo de fractura de cadera y del $58 \%$ de fracturas espinales ${ }^{7}$. La infección por Clostridioides difficile es otra de las asociaciones que se han hecho con el uso de IBP. Un incremento en el riesgo de infección por $C$. difficile, tanto incidental como recurrente, fue claramente demostrado en pacientes tratados con IBP ${ }^{8}$. Una de las asociaciones de mayor relevancia es la enfermedad renal aguda y crónica entre los pacientes usuarios de IBP, lo cual quedó de manifiesto en la prueba prospectiva de 10,482 participantes con función renal normal al inicio y con seguimiento de 13.9 años, revelando que los usuarios de IBP tenían un 50\% de mayor riesgo de desarrollar enfermedad renal crónica en comparación con quienes no tomaban este tipo de fármacos ${ }^{9}$. Recientemente se ha sugerido un incremento en el riesgo de demencia entre los usuarios de IBP. Haenisch, et al. ${ }^{10}$ estudiaron 3,076 pacientes mayores de 75 años sin historial previo de demencia, encontrando que el empleo de IBP aumentaba el riesgo de demencia en un $38 \%$ y en un $44 \%$ el de enfermedad de Alzheimer. 
Tabla 1. Indicaciones terapéuticas aprobadas por la FDA para el uso de IBP

Tratamiento de la enfermedad por reflujo gastroesofágico

- Resolución de esofagitis erosiva

- Tratamiento de mantenimiento de esofagitis erosiva resuelta

Tratamiento de ulceras gástricas o duodenales

Tratamiento y profilaxis de úlceras inducidas por AINE

Tratamiento para infección por Helicobacter pylori en combinación con antibióticos

Manejo de condiciones patológicas hipersecretoras (incluyendo el síndrome de Zollinger-Ellison)

FDA: Food and Drug Administration; IBP: inhibidores de la bomba de protones; AINE: antiinflamatorios no esteroideos.

Tabla 2. Riesgos asociados con el uso crónico de IBP

\begin{tabular}{|c|c|c|c|}
\hline Efecto adverso & $\begin{array}{c}\text { Riesgo relativo/ } \\
\text { odds ratio (IC 95\%) }\end{array}$ & $\begin{array}{l}\text { Calidad de } \\
\text { la evidencia }\end{array}$ & Recomendaciones prácticas \\
\hline \multicolumn{4}{|l|}{ Causa probable } \\
\hline Hipomagnesemia ${ }^{4}$ & $1.43(1.08-1.88)$ & Baja & Vigilar niveles de magnesio en pacientes sintomáticos \\
\hline Deficiencia de vitamina $B_{12}{ }^{5}$ & $1.65(1.58-1.73)$ & Baja & BH cada 2 años y vit. $B_{12}$ cada 5 años \\
\hline Sobrecrecimiento bacteriano $0^{6}$ & $2.28(1.24-4.21)$ & Baja & No hay recomendación para screening \\
\hline \multicolumn{4}{|l|}{ Asociación poco clara } \\
\hline Fracturas óseas ${ }^{7}$ & $1.33(1.15-1.54)$ & Baja & Densitometría ósea e \\
\hline Infección por Clostridioides difficile ${ }^{8}$ & $1.74(1.47-2.85)$ & Baja & ingesta de calcio y vitamina $D$ en CDR \\
\hline Enfermedad renal crónica ${ }^{9}$ & $1.50(1.14-1.96)$ & Muy baja & No hay recomendaciones (uso cuidadoso de antibióticos) \\
\hline Demencia $^{10}$ & $1.44(1.36-1.52)$ & Muy baja & Niveles de creatinina sérica anuales. No hay recomendaciones \\
\hline \multicolumn{4}{|l|}{ Causa improbable } \\
\hline Neumonía adquirida en la comunidad ${ }^{11}$ & $1.27(1.06-1.54)$ & Muy baja & No hay recomendaciones \\
\hline
\end{tabular}

IBP: inhibidores de la bomba de protones; IC: intervalo de confianza; CDR: cantidad diaria recomendada; BH: biometría hemática.

Por último, los pacientes que usaban IBP desarrollaron neumonía adquirida en la comunidad 4.5 veces más cuando se comparó con aquellos que nunca los tomaban ${ }^{11}$ (Tabla 2).

\section{EFECTOS ADVERSOS SEVEROS DE LA TERAPIA CON INHIBIDORES DE LA BOMBA DE PROTONES}

\section{Supresión de ácido con inhibidores de la bomba de protones y efectos adversos}

Algunos de los supuestos efectos adversos de los IBP están relacionados con la profunda supresión de la secreción de ácido gástrico. Con tal supresión, los microrganismos que habrían sido destruidos por el ácido gástrico pueden colonizar el tracto gastrointestinal superior y, de esta manera, alterar el microbioma ${ }^{12}$. Una vez bloqueada la producción de ácido gástrico, aquellos microrganismos patógenos ingeridos que habrían sido destruidos por el ácido gástrico podrían sobrevivir y causar infecciones. Esta inhibición de ácido podría influir también en la absorción de ciertas vitaminas (p. ej., vitamina $B_{12}$ ), minerales (p. ej., calcio y hierro) y de algunos fármacos (p. ej., ketoconazol y digoxina).
La elevación de la gastrina sérica es otro efecto potencial indeseable de la supresión de ácido gástrico por IBP. La gastrina liberada por las células $G$ en la mucosa glandular pilórica del antro estimula la secreción de ácido por las células parietales en el cuerpo gástrico y fundus. Cuando el ácido alcanza el antro, se activa un reflejo neuronal que estimula las células endocrinas tipo $D$ en la mucosa glandular pilórica para de esta manera producir somatostatina ${ }^{3}$. La somatostatina inhibe la liberación de gastrina de las células $\mathrm{G}$ adyacentes, suprimiendo la secreción de ácido de las células parietales. Cuando los IBP bloquean la producción de ácido necesario para inactivar la producción de gastrina, la secreción de esta continúa sin disminuir y los niveles séricos aumentan ${ }^{13}$. La gastrina es un factor de crecimiento que puede incrementar la proliferación de células ECL (enterochromaffin-like), tanto en metaplasia de Barrett como en el colon ${ }^{14-16}$. El uso crónico de IBP se ha relacionado con un aumento en los niveles séricos de cromogranina $A$, un potenciador del cúmulo de células $E C L^{16}$. Este incremento en el cúmulo de células ECL se ha propuesto como la explicación al fenómeno de «rebote de hipersecreción de ácido», teniendo en cuenta que la suspensión abrupta de IBP usados de manera crónica resulta en la producción de niveles de ácido gástrico superiores a aquellos presen- 


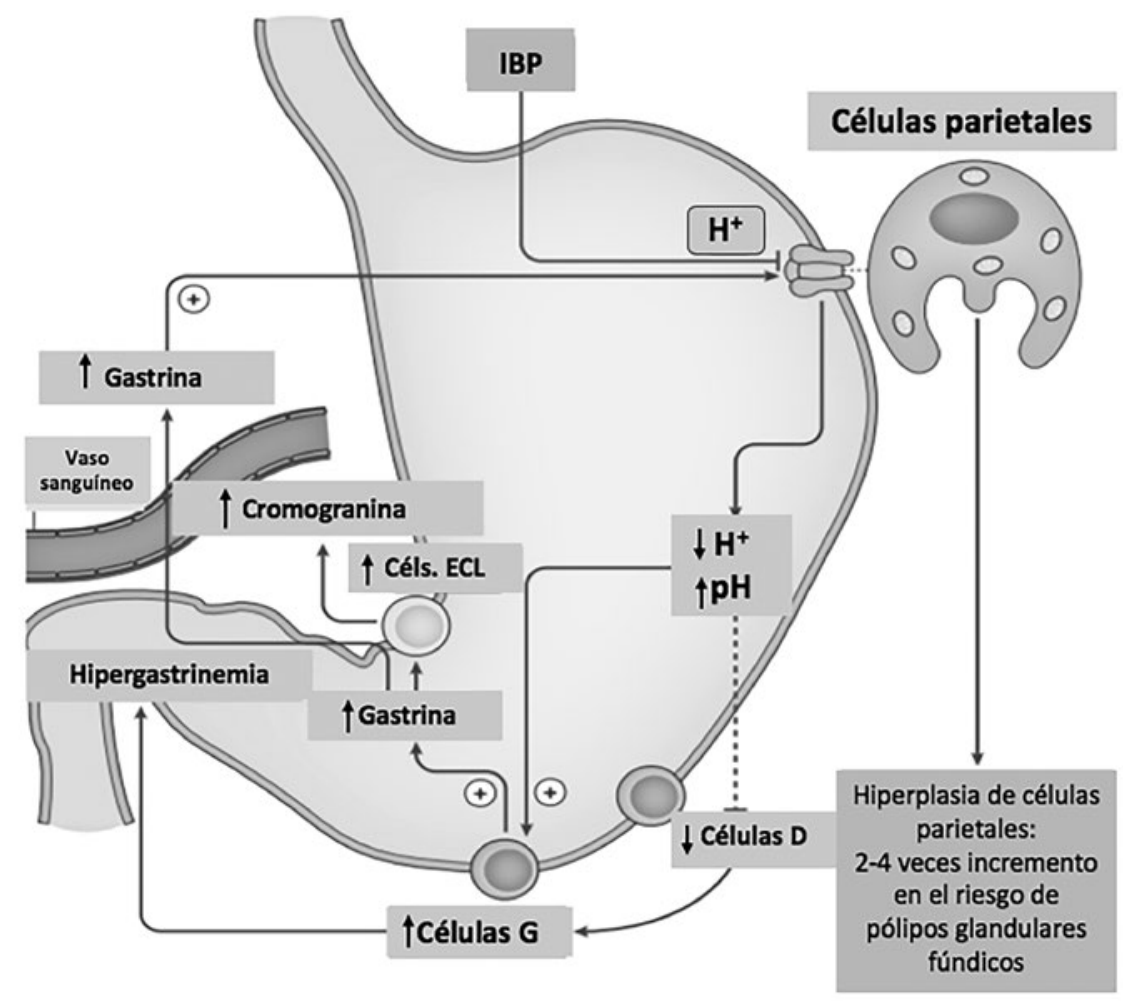

Figura 1. Efecto de los inhibidores de la bomba de protones (IBP) en la fisiología gástrica. La vía de alteración del ácido por los IBP, así como las supuestas vías, se muestran en líneas punteadas. La hipoclorhidria interfiere en las células $D$ y $G$ del antro dando como resultado un incremento en la gastrina y cromogranina, causando hiperplasia en las células ECL y células parietales. ECL: enterochromaffin-like.

tados antes del tratamiento con IBP ${ }^{17}$. Este rebote hipersecretor puede permanecer por meses después de la suspensión de IBP usados de manera crónica, sin embargo, su importancia clínica permanece incierta (Fig. 1).

\section{Categorías generales de los efectos adversos propuestos de los inhibidores de la bomba de protones}

Los efectos adversos propuestos de los IBP son cinco en general: a) se sabe que los IBP podrían incrementar el riesgo de desarrollar algunas neoplasias; b) se ha propuesto que los IBP incrementan el riesgo para desarrollar una gran variedad de infecciones; c) hay también propuestos varios riesgos de IBP relacionados con sus efectos sobre la absorción y metabolismo de ciertas vitaminas y minerales; d) los efectos de los IBP sobre la absorción y metabolismo de ciertos fármacos posiblemente podrían tener consecuencias adversas, y e) hay un número creciente de efectos misceláneos adversos propuestos de los IBP.

\section{Aumento en el riesgo de neoplasias}

Como se mencionó previamente, la gastrina puede incrementar la proliferación de células ECL. Está, además, el hallazgo prematuro de que ratones hembra tratadas por un largo periodo con omeprazol desarrollaron hipergastrinemia, hiperplasia de células ECL y tumores carcinoides gástricos (neuroendocrinos), hallazgos que fueron los causantes del retraso en EE.UU. en el lanzamiento del omeprazol ${ }^{18}$. La preocupación anterior fue disipada por estudios posteriores que mostraron que otros animales diferentes a las ratas hembra no desarrollaron tumores carcinoides con el tratamiento con IBP, así como que la hipergastrinemia inducida por IBP en humanos generalmente es leve y, aun siendo severa, rara vez causa tumores carcinoides en pacientes humanos a menos que existan anormalidades genéticas tales como neoplasia endocrina múltiple tipo 19-21. De igual manera, aunque la gastrina incrementa la proliferación de células ECL en el colon, los estudios no han encontrado un incremento en la frecuencia de cáncer de colon en pacientes tomando IBP ${ }^{22,23}$. 
Por diversas circunstancias, se ha propuesto que los IBP pudieran ser un factor de riesgo de cáncer en el esófago de Barrett, así como gástricos. Se ha demostrado que la gastrina aumenta la proliferación de metaplasia de Barrett y dicho efecto podría promover el desarrollo neoplásico ${ }^{15}$. La gastrina también incrementa la proliferación de células ECL, y datos recientes sugieren que algunos adenocarcinomas gástricos podrían originarse a partir de estas células $\mathrm{ECL}^{24}$. Así también, la supresión de ácido gástrico puede permitir la colonización gástrica por bacterias que son capaces de desconjugar ácidos biliares y, si estos refluyen hacia el esófago, pueden dañar la mucosa y así causar daño en el ADN25. Estas bacterias son capaces también de convertir los nitratos ingeridos en la dieta en compuestos nitrosos potencialmente carcinogénicos ${ }^{26}$. Por último, algunos estudios han sugerido que los IBP pueden acelerar el desarrollo de atrofia gástrica en pacientes infectados con Helicobacter pylori ${ }^{27}$. Aun cuando todos estos factores podrían promover la carcinogénesis, solo unos cuantos estudios han podido relacionar el uso de IBP con el desarrollo de adenocarcinomas esofágicos y gástri$\cos ^{28-30}$. Además, existen razones suficientes para pensar que los IBP podrían proteger contra el cáncer en esófago de Barrett. La inflamación crónica es un factor de riesgo para el desarrollo de cáncer en muchos órganos y, en este caso, los IBP mitigan dicha inflamación esofágica crónica causada por la esofagitis por reflujo. De igual forma, el ácido puede causar daño al ADN potencialmente carcinogénico en esófago de Barrett ${ }^{31}$, y los IBP disminuyen la exposición esofágica al ácido. Aunado a esto y aunque no todos los estudios en esta área sugieren que los IBP protegen contra el cáncer en esófago de Barrett 32,33 , concluyen que los efectos protectores de los IBP, hablando de cáncer, superan su potencial para promover cáncer en esta condición.

\section{Aumento en el riesgo de infecciones}

El ácido gástrico juega un papel importante en la fagocitosis de microrganismos ingeridos y existen reportes sugiriendo que la supresión del ácido con IBP puede incrementar el riesgo de contraer infecciones intestinales por microorganismos tales como Salmonella y Campylobacter ${ }^{34}$, neumonía adquirida en la comunidad (debido a la colonización gástrica con bacterias que en presencia de reflujo podrían ser broncoaspiradas) $)^{35}$, infecciones por $C$. difficile ${ }^{36}$, así como peritonitis bacteriana espontánea en pacientes con cirrosis (debido a alteraciones en el microbioma inducida por IBP, facilitando la traslocación de bacterias intestinales hacia el líquido ascítico $)^{36}$.

\section{Absorción de vitaminas, minerales y efectos metabólicos}

Los IBP pueden interferir con la absorción de vitamina $\mathrm{B}_{12}$, hierro y calcio. La actividad ácido péptica es indispensable para liberar la cobalamina unida a proteínas en la dieta para que de esta manera se pueda unir al factor intrínseco y posteriormente absorberse en el íleon terminal, existiendo reportes de pacientes usuarios crónicos de IBP que desarrollan deficiencia de vitamina $B_{12}{ }^{37}$. El ácido gástrico facilita la oxidación de hierro férrico en la dieta a hierro ferroso para su absorción en el duodeno, habiendo reportes de anemia por deficiencia de hierro en pacientes usuarios de IBP38. El ácido gástrico también facilita la absorción de calcio induciendo la ionización y solubilización de sales de calcio, y recientemente se ha propuesto que los IBP pudieran incrementar el riesgo de osteoporosis, así como de fracturas óseas ${ }^{39,40}$. De igual manera se ha asociado al uso de IBP la hipomagnesemia, aunque no se saben con exactitud los mecanismos fisiopatológicos implicados ${ }^{41}$.

\section{Absorción y metabolismo de fármacos}

La supresión de ácido gástrico causada por los IBP influye sobre la absorción de algunos fármacos, con algunas excepciones importantes. Por ejemplo, el ketoconazol puede no absorberse de manera adecuada en ausencia del ácido gástrico y, por otro lado, en presencia de $\mathrm{pH}$ gástrico elevado se facilita la absorción de digoxina ${ }^{42}$. Los IBP se metabolizan a través del sistema del citocromo P450 y, así, podrían afectar el metabolismo de otros fármacos que lo hagan a través de esta vía ${ }^{43}$. Los IBP también pueden retrasar el aclaramiento del diazepam, la warfarina y la fenitoína, teniendo en cuenta que son raros los efectos adversos clínicamente relevantes ${ }^{44}$. El potencial de los IBP para reducir la acción antiplaquetaria del clopidogrel ha sido de gran relevancia en los últimos años, ya que ambos, tanto los IBP como el clopidogrel, son metabolizados por la enzima CYP2C $19^{45} \mathrm{y}$, por consecuencia, disminuye el efecto antiplaquetario ${ }^{46}$. Debido a todo lo anterior, la FDA (Food and Drug Administration) ha emitido varias alertas sobre el uso concomitante de omeprazol 
o esomeprazol con clopidogrel. Sin embargo, varios estudios controlados aleatorizados no han encontrado diferencias significativas en los resultados cardiovasculares en pacientes usando clopidogrel con o sin omeprazol ${ }^{47}$. Desafortunadamente, ninguno de estos estudios fue lo suficientemente contundente para excluir una pequeña pero potencialmente importante diferencia en la frecuencia de eventos cardiovasculares debido al uso de IBP.

\section{Efectos misceláneos}

Algunos efectos adversos misceláneos se han incrementado considerablemente de manera reciente. Estos incluyen infarto agudo de miocardio, evento vascular cerebral isquémico, enfermedad renal aguda y crónica, colitis microscópica, esofagitis eosinofílica, enfermedad celiaca, demencia y muerte.

Si bien se han descrito efectos adversos y aumento de eventos cardiovasculares en pacientes tomando clopidogrel junto con IBP, inicialmente explicado por la competencia en el metabolismo del CYP2C19, existen ensayos clínicos de pacientes con síndrome coronario agudo tratados con ticagrelor, un antiplaquetario que no es metabolizado a través de la vía del CYP2C19, y también se encontró un incremento en la frecuencia de eventos adversos cardiovasculares en pacientes tomando IBP de manera concomitante ${ }^{48}$. Existe una explicación propuesta para este efecto que involucra efectos complicados de los IBP que resultan en disminución en la producción de óxido nítrico, alterando la homeostasis vascular ${ }^{49}$.

Existen más de 250 fármacos implicados en el desarrollo de nefritis intersticial aguda, siendo esta una reacción de hipersensibilidad idiosincrática, entre los cuales se encuentran los IBP50. La nefritis intersticial aguda puede causar daño renal severo progresando a enfermedad renal crónica. El uso de IBP se ha asociado con enfermedad renal crónica, aunque no está claro si la nefritis intersticial aguda es el mecanismo precursor en todos los $\operatorname{casos}^{51,52}$

Se ha propuesto que los IBP pueden contribuir al desarrollo de esofagitis eosinofílica debido a sus efectos sobre la digestión péptica, así como la permeabilidad gástrica ${ }^{53}$. Los IBP aumentan el pH gástrico a niveles en los cuales la pepsina no es activa $(\mathrm{pH}>4) \mathrm{y}$, por consecuencia, los péptidos ingeridos en la dieta que no son degradados por la pepsina en la cámara gástrica pueden provocar una respuesta alérgica cuando llegan al intestino delgado. Además, Ios IBP incrementan la permeabilidad de la mucosa gástrica y de esta manera se facilita la absorción de alergenos peptídicos no degradados con la consecuente exposición de células inmunitarias. Los efectos antes mencionados sobre el microbioma intestinal podrían predisponer al desarrollo de esofagitis eosinofílica. Existen mecanismos similares que pueden alterar la degradación e inmunogenicidad del gluten de la dieta, siendo esta una explicación de la asociación de IBP con enfermedad celiaca ${ }^{54}$. De igual manera, dichos mecanismos también se han asociado al desarrollo de colitis microscópica ${ }^{55}$. El papel de Ios IBP en el desarrollo de esofagitis eosinofílica es paradójico, ya que estos se utilizan en su tratamiento. Sin embargo, los mecanismos propuestos que explican los efectos benéficos de los IBP en la esofagitis eosinofílica (bloqueo de la producción de eotaxina-3 estimulada por citocinas TH2) son diferentes a los propuestos en la patogénesis de esofagitis eosinofílica ${ }^{56}$.

Se cree que la acumulación de $\beta$-amiloide a nivel cerebral juega un papel importante en el desarrollo de enfermedad de Alzheimer. Se ha propuesto que los IBP pudieran ser un factor de riesgo para el desarrollo de demencia debido a que dificultan la degradación de $\beta$-amiloide, previniendo la acidificación de los lisosomas microgliales ${ }^{57}$. Por último, el uso crónico de IBP se ha asociado con un aumento en el riesgo de muerte por todas las causas; sin embargo, dicha asociación no está clara, ya que se desconocen los mecanismos que contribuyen a este riesgo ${ }^{58}$ (Fig. 2).

\section{CONCLUSIONES}

Los IBP se encuentran dentro de los fármacos más seguros y efectivos; sin embargo, los datos recientes muestran una gran cantidad de efectos adversos. La gran mayoría de estas asociaciones se basan en estudios observacionales, existiendo factores confusionales, por lo que es necesario dar posibles explicaciones alternativas al momento de evaluar estos estudios. En la mayoría de los casos, los estudios controlados aleatorizados serían de gran utilidad para confirmar estas asociaciones, teniendo en cuenta la complejidad para su realización. 


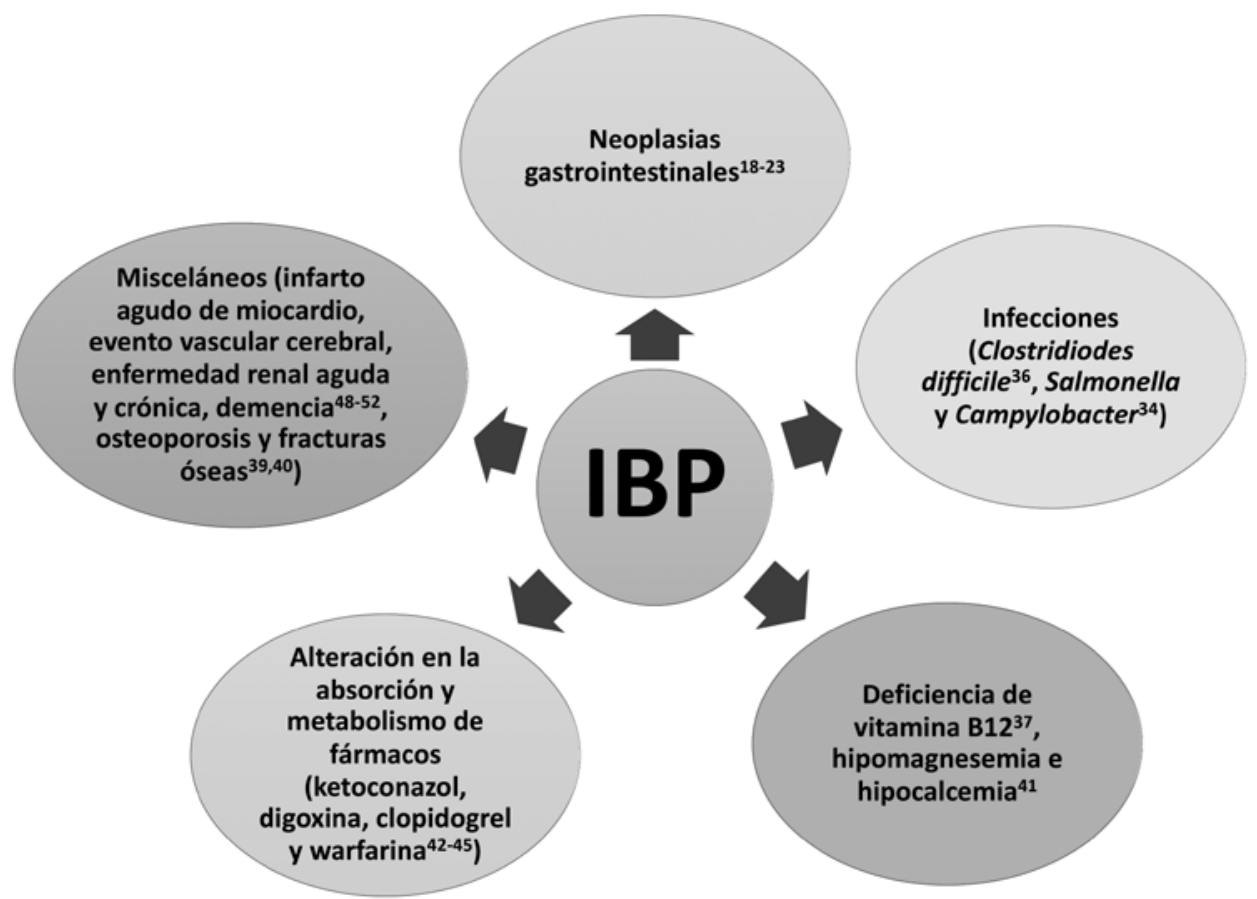

Figura 2. Efectos adversos de los inhibidores de bomba de protones (IBP).

Gran parte de estos efectos secundarios podría explicarse a través de mecanismos biológicos subyacentes claros. Sin embargo, la evidencia clínica de estos efectos adversos es generalmente débil y no puede ser completamente asociada al uso de IBP. En especial, dado que los esquemas cortos de tratamiento con IBP no representan algún peligro, se debe alertar y poner gran atención en pacientes geriátricos en tratamiento a largo plazo con IBP que, además, tengan comorbilidades y polifarmacia en el momento de prescribirlos. Por último, en la mayoría de los casos, y basados en la evidencia actual, los beneficios de los IBP parecen superar a los efectos adversos. Sin embargo, los IBP se prescriben con frecuencia de manera indiscriminada; por ello se deben revisar siempre las indicaciones clínicas para determinar si el tratamiento es realmente necesario.

\section{FINANCIAMIENTO}

Los autores no recibieron patrocinio para llevar a cabo este artículo

\section{CONFLICTO DE INTERESES}

Los autores declaran no tener conflictos de intereses.

\section{RESPONSABILIDADES ÉTICAS}

Protección de personas y animales. Los autores declaran que para esta investigación no se han realizado experimentos en seres humanos ni en animales.

Confidencialidad de los datos. Los autores declaran que en este artículo no aparecen datos de pacientes.

Derecho a la privacidad y consentimiento informado. Los autores declaran que en este artículo no aparecen datos de pacientes.

\section{BIBLIOGRAFÍA}

1. Naunton M, Peterson GM, Deeks LS, Young H, Kosari S. We have had a gutful: the need for deprescribing proton pump inhibitors. J Clin Pharm Ther. 2018;43:65-72.

2. Kantor ED, Rehm CD, Haas JS, Chan AT, Giovannucci EL. Trends in prescription drug use among adults in the United States from 1999-2012. JAMA. 2015;314:1818-31.

3. Nehra AK, Alexander JA, Loftus CG, Nehra V. Proton pump inhibitors: Review of emerging concerns. Mayo Clin Proc. 2018;93(2):240-6.

4. Cheungpasitporn W, Thongprayoon C, Kittanamongkolchai W, Srivali N, Edmonds PJ, Ungprasert P, et al. Proton pump inhibitors linked to hypomagnesemia: a systematic review and meta-analysis of observational studies. Ren Fail. 2015;37(7):1237-41.

5. Lam JR, Schneider JL, Zhao W, Corley DA. Proton pump inhibitor and histamine 2 receptor antagonist use and vitamin B 12 deficiency. JAMA. 2013;310(22):2435-42.

6. Lo WK, Chan WW. Proton pump inhibitor use and the risk of small intestinal bacterial overgrowth: a meta-analysis. Clin Gastroenterol Hepatol. 2013;11(5):483-90. 
7. Zhou B, Huang Y, Li H, Sun W, Liu J. Proton-pump inhibitors and risk of fractures: an update meta-analysis. Osteoporos Int. 2016;27(1):339-47.

8. Kwok CS, Arthur AK, Anibueze Cl, Singh S, Cavallazzi R, Loke YK. Risk of Clostridium difficile infection with acid suppressing drugs and antibiotics: meta-analysis. Am J Gastroenterol. 2012;107(7):1011-9.

9. Lazarus B, Chen Y, Wilson FP, Sang Y, Chang AR, Coresh J, et al. Proton pump inhibitor use and the risk of chronic kidney disease. JAMA Intern Med. 2016:176(2):238-46

10. Haenisch B, von Holt K, Wiese B, Prokein J, Lange C, Ernst A, et al. Risk of dementia in elderly patients with the use of proton pump inhibitors. Eur Arch Psychiatry Clin Neurosci. 2015;265(5):419-28.

11. Laheij RJ, Sturkenboom MC, Hassing RJ, Dieleman J, Stricker BH, Janse JB. Risk of community-acquired pneumonia and use of gastric acid-suppressive drugs. JAMA. 2004;292(16):1955-60.

12. Naito $\mathrm{Y}$, Kashiwagi $\mathrm{K}$, Takagi T, Andoh A, Inoue R. Intestinal dysbiosis secondary to proton pump inhibitor use. Digestion. 2018;97:195-204.

13. Lundell L, Vieth M, Gibson F, Nagy P, Kahrilas PJ. Systematic review: the effects of long-term proton pump inhibitor use on serum gastrin levels and gastric histology. Aliment Pharmacol Ther. 2015;42:649-63.

14. Dockray GJ, Varro A, Dimaline R, Wang T. The gastrins: their production and biological activities. Annu Rev Physiol. 2001;63:119-39.

15. Haigh CR, Attwood SE, Thompson DG, Jankowski JA, Kirton CM, Pritchard $\mathrm{DM}$, et al. Gastrin induces proliferation in Barrett's metaplasia through activation of the CCK2 receptor. Gastroenterology. 2003;124:615-25.

16. Waldum HL, Arnestad JS, Brenna E, Eide I, Syversen U, Sandvik AK. Marked increase in gastric acid secretory capacity after omeprazole treatment. Gut. 1996;39:649-53.

17. Fossmark R, Johnsen G, Johanessen E, Waldum HL. Rebound acid hypersecretion after long-term inhibition of gastric acid secretion. Aliment Pharmacol Ther. 2005;21:149-54.

18. Havu N. Enterochromaffin-like cell carcinoids of gastric mucosa in rats after lifelong inhibition of gastric secretion. Digestion. 1986;35(Suppl 1):42-55.

19. Freston JW. Clinical significance of hypergastrinaemia: relevance to gastrin monitoring during omeprazole therapy. Digestion. 1992;51(Suppl 1): 102-14.

20. Solcia E, Capella C, Fiocca R, Rindi G, Rosai J. Gastric argyrophil carcinoidosis in patients with Zollinger-Ellison syndrome due to type 1 multiple endocrine neoplasia. A newly recognized association. Am J Surg Pathol. 1990;4:503-13.

21. Laine L, Ahnen D, McClain C, Solcia E, Walsh JH. Review article: potential gastrointestinal effects of long-term acid suppression with proton pump inhibitors. Aliment Pharmacol Ther. 2000:14:651-8.

22. Singh M, Dhindsa G, Friedland S, Triadafilopoulos G. Long-term use of proton pump inhibitors does not affect the frequency, growth, or histologic characteristics of colon adenomas. Aliment Pharmacol Ther. 2007; 26:1051-61.

23. Ahn JS, Park SM, Eom CS, Kim S, Myung SK. Use of proton pump inhibitor and risk of colorectal cancer: a meta-analysis of observational studies. Korean J Fam Med. 2012;33:272-9.

24. Waldum HL, Sørdal $\emptyset$, Fossmark R. Proton pump inhibitors (PPIs) may cause gastric cancer - clinical consequences. Scand J Gastroenterol. 2018;53(6):639-42.

25. Theisen J, Nehra D, Citron D, Johansson J, Hagen JA, Crookes PF, et al. Suppression of gastric acid secretion in patients with gastroesophageal reflux disease results in gastric bacterial overgrowth and deconjugation of bile acids. J Gastrointest Surg. 2000:4:50-4.

26. Calmels S, Béréziat JC, Ohshima $\mathrm{H}$, Bartsch $\mathrm{H}$. Bacterial formation of $\mathrm{N}$ nitroso compounds from administered precursors in the rat stomach after omeprazole induced achlorhydria. Carcinogenesis. 1991:12:435-9.

27. Kuipers EJ, Lee A, Klinkenberg-Knol EC, Meuwissen SG. Review article: the development of atrophic gastritis-Helicobacter pylori and the effects of acid suppressive therapy. Aliment Pharmacol Ther. 1995;9:331-40.

28. Cheung KS, Chan EW, Wong AYS, Chen L, Wong ICK, Leung WK. Long-term proton pump inhibitors and risk of gastric cancer development after treatment for Helicobacter pylori: a population-based study. Gut. 2018;67:28-35.

29. Brusselaers N, Wahlin K, Engstrand L, Lagergren J. Maintenance therapy with proton pump inhibitors and risk of gastric cancer: a nationwide population-based cohort study in Sweden. BMJ Open. 2017;7:e017739.

30. Brusselaers N, Engstrand L, Lagergren J. Maintenance proton pump inhibition therapy and risk of oesophageal cancer. Cancer Epidemiol. 2018;53: 172-7.

31. Zhang HY, Hormi-Carver K, Zhang X, Spechler SJ, Souza RF. In benign Barrett's epithelial cells, acid exposure generates reactive oxygen species that cause DNA double strand breaks. Cancer Res. 2009;69:9083-9.

32. Singh S, Garg SK, Singh PP, Iyer PG, El-Serag HB. Acid-suppressive medications and risk of oesophageal adenocarcinoma in patients with Barrett's oesophagus: a systematic review and meta-analysis. Gut. 2014:63:1229-37.

33. Hu Q, Sun TT, Hong J, Fang JY, Xiong H, Meltzer SJ, et al. Proton pump inhibitors do not reduce the risk of esophageal adenocarcinoma in patients with Barrett's esophagus: a systematic review and meta-analysis. PLOS One. 2017;12(1):e0169691.
34. Bavishi C, Dupont HL. Systematic review: the use of proton pump inhibitors and increased susceptibility to enteric infection. Aliment Pharmacol Ther. 2011;34:1269-81.

35. Lambert AA, Lam JO, Paik JJ, Ugarte-Gil C, Drummond MB, Crowell TA. Risk of community-acquired pneumonia with outpatient proton-pump inhibitor therapy: a systematic review and meta-analysis. PLOS One. 2015; 10:e0128004.

36. Deshpande A, Pasupuleti V, Thota P, Pant C, Mapara S, Hassan S, et al. Acid-suppressive therapy is associated with spontaneous bacterial peritonitis in cirrhotic patients: a meta-analysis. J Gastroenterol Hepatol. 2013:28:235-42

37. Lam JR, Schneider JL, Zhao W, Corley DA. Proton pump inhibitor and histamine 2 receptor antagonist use and vitamin B12 deficiency. JAMA. 2013:310:2435-42.

38. Sarzynski E, Puttarajappa C, Xie Y, Grover M, Laird-Fick H.Association between proton pump inhibitor use and anemia: a retrospective cohort study. Dig Dis Sci. 2011;56:2349-53.

39. Andersen BN, Johansen PB, Abrahamsen B. Proton pump inhibitors and osteoporosis. Curr Opin Rheumatol. 2016;28:420-5.

40. Ngamruengphong S, Leontiadis GI, Radhi S, Dentino A, Nugent K.Proton pump inhibitors and risk of fracture: a systematic review and meta-analysis of observational studies. Am J Gastroenterol. 2011:106:1209-18.

41. William JH, Danziger J. Proton-pump inhibitor-induced hypomagnesemia: current research and proposed mechanisms. World J Nephrol. 2016:5:152-7.

42. Lew EA. Review article: pharmacokinetic concerns in the selection of antiulcer therapy. Aliment Pharmacol Ther. 1999;13(suppl 5):11-6.

43. VandenBranden M, Ring BJ, Binkley SN, Wrighton SA. Interaction of human liver cytochromes P450 in vitro with LY307640, a gastric proton pump inhibitor. Pharmacogenetics. 1996;6:81-91.

44. Humphries TJ, Merritt GJ. Review article: drug interactions with agents used to treat acid-related diseases. Aliment Pharmacol Ther. 1999:13(Suppl 3):18-26.

45. Gilard M, Arnaud B, Le Gal G, Abgrall JF, Boschat J. Influence of omeprazole on the antiplatelet action of clopidogrel associated to aspirin. J Thromb Haemost. 2006:4:2508-9.

46. Gilard M, Arnaud B, Cornily JC, Le Gal G, Lacut K, Le Calvez G, et al. Influence of omeprazole on the antiplatelet action of clopidogrel associated with aspirin: the randomized, double-blind OCLA (Omeprazole Clopidogrel Aspirin) study. J Am Coll Cardiol. 2008;51:256-60.

47. Melloni C, Washam JB, Jones WS, Halim SA, Hasselblad V, Mayer SB, et al. Conflicting results between randomized trials and observational studies on the impact of proton pump inhibitors on cardiovascular events when coadministered with dual antiplatelet therapy: systematic review. Circ Cardiovasc Qual Outcomes. 2015;8:47-55.

48. Goodman SG, Clare R, Pieper KS, Nicolau JC, Storey RF, Cantor WJ, et al. Platelet Inhibition and Patient Outcomes Trial Investigators. Association of proton pump inhibitor use on cardiovascular outcomes with clopidogrel and ticagrelor: insights from the platelet inhibition and patient outcomes trial. Circulation. 2012:125:978-86.

49. Ghebremariam YT, LePendu P, Lee JC, Erlanson DA, Slaviero A, Shah NH, et al. Unexpected effect of proton pump inhibitors: elevation of the cardiovascular risk factor asymmetric dimethylarginine. Circulation. 2013;128: 845-53.

50. Raghavan R, Shawar S. Mechanisms of drug-induced interstitial nephritis. Adv Chronic Kidney Dis. 2017:24:64-71

51. Yang Y, George KC, Shang WF, Zeng R, Ge SW, Xu G. Proton pump inhibitors use, and risk of acute kidney injury: a meta-analysis of observational studies. Drug Des Devel Ther. 2017;11:1291-9.

52. Nochaiwong S, Ruengorn C, Awiphan R, Koyratkoson K, Chaisai C, Noppakun $\mathrm{K}$, et al. The association between proton pump inhibitor use and the risk of adverse kidney outcomes: a systematic review and meta-analysis. Nephrol Dial Transplant. 2018:33:331-42.

53. Merwat SN, Spechler SJ. Might the use of acid suppressive medications predispose to the development of eosinophilic esophagitis? Am J Gastroenterol. 2009;104:1897-902

54. Lebwohl B, Spechler SJ, Wang TC, Green PHR, Ludvigsson JF. Use of proton pump inhibitors and subsequent risk of celiac disease. Dig Liver Dis. 2014;46:36-40

55. Law EH, Badowski M, Hung YT, Weems K, Sanchez A, Lee TA. Association between proton pump inhibitors and microscopic colitis. Ann Pharmacother. 2017;51:253-63

56. Cheng E, Zhang X, Huo X, Yu C, Zhang Q, Wang DH, et al. Omeprazole blocks eotaxin-3 expression by oesophageal squamous cells from patients with eosinophilic oesophagitis and GORD. Gut. 2013;62:824-32.

57. Fallahzadeh MK, Borhani Haghighi A, Namazi MR. Proton pump inhibitors: predisposers to Alzheimer disease? J Clin Pharm Ther. 2010;35: 125-6.

58. Xie Y, Bowe B, Li T, Xian H, Yan Y, Al-Aly Z.Risk of death among users of proton pump inhibitors: a longitudinal observational cohort study of United States veterans. BMJ Open. 2017;7(6):e015735. 